\title{
The Relation between Acculturation and Psychological Well-Being among Adolescents of Asian Origin
}

\author{
Yong $\mathrm{Li}^{1}$, Yuqi Guo ${ }^{2}$ \\ ${ }^{1}$ Department of Social Work, California State University, Bakersfield, United States \\ ${ }^{2}$ School of Social Work, University of Alabama, United States \\ Correspondence: Yong Li, Department of Social Work, California State University, Bakersfield, United States.
}

Received: January 19, 2018

doi:10.11114/ijsss.v6i4.2938

\author{
Accepted: February 7, $2018 \quad$ Available online: February 23, 2018 \\ URL: https://doi.org/10.11114/ijsss.v6i4.2938
}

\begin{abstract}
The current study aimed to test the direct and indirect effects of acculturation on the psychological well-being among the adolescent children of Asian origin. Subgroup analysis was conducted to compare these effects between Asian refugee and non-refugee children. Data were from the follow-up survey of the Children of Immigrants Longitudinal Study (CILS). The analytic sample included adolescents from Asian refugee families $(\mathrm{N}=563)$ and non-refugee families $(\mathrm{N}=779)$. Adolescent acculturation was assessed by three proxy measures: nativity, time in the United States, and U.S. preference. Family dynamics were measured by adolescents' perceptions about parent-child conflict and family cohesion. Multi-group structural equation modeling was used to analyze the data. Findings suggested that the direct effects of acculturation on psychological well-being were dependent upon the proxy measure of acculturation and the immigration type. Mediation analyses showed that higher level of acculturation was generally related to higher parent-child conflict and lower family cohesion, which, in turn, were associated with lower psychological well-being in the refugee subsample, but not the non-refugee subsample. Our findings call for programs that can take advantage of the protective role of family cohesion and reduce the negative impact of parent-child conflict. Special attention needs to be paid to the refugee population from Asia.
\end{abstract}

Keywords: children of immigrants, Southeast Asian refugees, acculturation, family dynamics, psychological well-being

\section{Introduction}

Although children of Asian immigrants are often portrayed as the "model minority", they are faced with challenges including parent-child conflict, poor peer relationships, depression, and low self-esteem (Huang, Calzada, Cheng, \& Brotman, 2012; Kiang, Tseng, \& Yip, 2016). Moreover, outcomes in psychological and sociocultural adaption may differ across ethnic groups of Asian immigrants, given the variation in history, culture, and migration experience (e.g., Kim, Yang, Atkinson, Wolfe, \& Hong, 2001). Southeast Asian refugees may face more psychological problems (e.g., PTSD, depression, and anxiety) and socioeconomic hardships after immigration due to the traumatic refugee experiences and limited English proficiency (Le \& Nguyen, 2012). Consequently, their children may be more likely to be involved in gang activities, drop out of school, experience depression, and engage in violent behaviors (Han \& Lee, 2011; Ngo \& Lee, 2007). As two indicators of psychological well-being, self-esteem and depressive symptoms have been found to be highly related to risk behaviors such as substance use, early sexual activity, smoking, and suicide among Asian adolescents (e.g., Rhee, Chang, \& Rhee, 2003; Rosario-Sim \& O'Connell, 2009), as well as adolescents in the general population (e.g., Diego, Field, \& Sanders, 2003; Isomaa, Väänänen, Fröjd, Kaltiala-Heino, \& Marttunen, 2013; Spirito \& Esposito-Smythers, 2006).

Acculturation has been linked to the psychological well-being of children in Asian immigrant families (e.g., Hwang \& Ting, 2008; Rosario-Sim \& O'Connell, 2009). Another line of research has focused on the impact of family factors on the psychological well-being of children of Asian immigrants. Of particular interest is the role of parent-child conflict (e.g., Cheng, Lin, \& Cha, 2015; Lui, 2015) and family cohesion (e.g., Juang \& Alvarez, 2010; Li \& Warner, 2015). Despite these advances, the interrelationships between acculturation and family factors and the comparison between children of Southeast Asian refugee families and children of non-refugee Asian families remain understudied.

In this study, Asian immigrants are loosely defined as immigrants of all Asian backgrounds which include both the refugee subgroup and the non-refugee subgroup. For the purpose of this study, the refugee subgroup includes Asians of 
Vietnamese, Cambodian, Hmong, and Laotian origin; the non-refugee subgroup includes Asians of Filipino and Chinese origin. Children of Asian origin (i.e., Asian immigrants) are defined as adolescents under age 18 who have at least one Asian immigrant parent. Finally, children and adolescents are used interchangeably. Using a sample of children of Asian immigrants, the current study aimed to: 1) examine the direct associations between adolescent acculturation and psychological well-being (i.e., self-esteem and depressive symptoms); 2) test the indirect effects of acculturation on psychological well-being via family dynamics (including parent-child conflict and family cohesion); and 3) compare these effects between Asian refugee and non-refugee adolescents.

\subsection{The Direct Effect of Acculturation on Adolescent Psychological Well-Being}

Traditionally, acculturation was understood as a process in which an immigrant adapts to the receiving culture and eventually loses the heritage culture (Gordon, 1964). Thus, the process is linear and the two cultures are mutually exclusive. More recently, researchers have begun to understand acculturation from a bidimensional perspective and argue that maintenance of traditional culture and endorsement of dominant culture coexist with one another (e.g., De La Rosa, 2002). Nevertheless, researchers still follow the unidimensional tradition and study acculturation in terms of its "level" or "degree" (Berry, 2003, p.22). For example, acculturation level has been operationally defined as language fluency, social affiliation, cultural knowledge, and/or daily living preferences, as well as other proxy measures such as nativity and time living in the host county (Lara, Gamboa, Kahramanian, Morales, \& Bautista, 2005).

This study employed the unidimensional definition of acculturation (i.e., acculturation level). Empirical studies using acculturation proxies have reported conflicting findings on the relationship between acculturation and the psychological well-being among Asian immigrant adolescents. On the one hand, Hwang \& Ting (2008) reported that higher levels of acculturation were associated with lower psychological distress and clinical depression among Chinese, Japanese, Korean, and Vietnamese American college students. Similarly, Yeh (2003) found an inverse association between acculturation level and a variety of mental health symptoms among an ethnically diverse group of Asian adolescents. One explanation could be that more acculturated adolescents experienced lower acculturative stress, which in turn, was related to fewer mental health problems (Organista, Organista, \& Kurasaki, 2003).

On the other hand, acculturation levels have been found to be a risk factor. Researchers have reported that higher levels of acculturation were associated with more social adjustment difficulties such as negative self-images and low self-esteem among adolescents of Chinese, Japanese, Korean, Vietnamese, and Indian backgrounds (Rhee, Chang, \& Rhee, 2003). Similarly, Rosario-Sim \& O'Connell (2009) reported that being more acculturated (measured by English proficiency) was associated with smoking cigarettes among a diverse sample of Asian American adolescents in New York City.

\subsection{The Indirect Effects of Acculturation Through Family Dynamics}

Studying the mediating factors between acculturation and psychological well-being is critical because these mediators play a more proximal role. In order to improve the psychological well-being of children of immigrants, professionals who work with this population may need to target the mediators instead of acculturation, especially when the effect of acculturation is inconclusive. Few previous empirical studies have examined the indirect effects of acculturation on child psychological well-being through family dynamics. One exception is Birman \& Taylor-Ritzler's (2007) study on immigrant adolescents from the former Soviet Union resettled in the United States. They used support from parents, satisfaction with parents, and family cohesion to measure family relationships as a latent construct and found that family relationships partially mediated the link between adolescent acculturation and psychological distress. In their study, family cohesion was defined as "the degree of commitment, help, and support family members provide for one another" (Birman \& Taylor-Ritzler, 2007, p.342). In addition, other researchers have considered the role of parent-child conflict in shaping psychological well-being of adolescents in immigrant families. Parent-child conflict may be a result of the developmental stage of adolescents and, more importantly, acculturation of adolescents in immigrant families $(\mathrm{Li}$, 2013). The following review focuses on parent-child conflict and family cohesion as two potential mediators.

Acculturation seems to have a direct impact on parent-child conflict in Asian families. For example, more acculturated Vietnamese young adults were reported to experience higher parent-child conflict (Vu \& Rook, 2013). It has also been suggested that adolescent acculturation affected family cohesion in immigrant families (Marsiglia, Parsai, \& Kulis, 2009). For example, researchers (Baer \& Schmitz, 2007) have reported that more acculturated adolescents of Mexican origin experienced lower family cohesion than their less acculturated counterparts.

Meanwhile, studies on the direct relationships between family dynamics and psychological well-being are plentiful. Evidence indicates that parent-child conflict was a risk factor for various psychological and behavioral problems among youth of diverse Asian backgrounds (e.g., Choi, He, \& Harachi, 2008; Lui, 2015; Wyatt, Ung, Park, Kwon, \& Trinh-Shevrin, 2015; Ying \& Han, 2007). As suggested by a recent meta-analysis study, this held true for different type of conflict and youth outcomes (Weymouth, Buehler, Zhou, \& Henson, 2016). In addition, family cohesion has been 
found to be a protective factor for the mental health of both Asian and Latino American immigrants (Leong, Park, \& Kalibatseva, 2013). Taken together, these findings converge on the notion that higher acculturation may predict higher parent-child conflict and lower family cohesion, which in turn endangers the psychological well-being among Asian American youth.

\subsection{Comparison between Asian Refugee and Non-Refugee Families}

It is important to study different Asian subgroups given the vast diversity (such as country of origin and ethnicity) in Asian immigrants (Kalibatseva \& Leong, 2011). However, Kalibatseva \& Leong (2011) also acknowledged the practical pitfalls in collecting large enough samples of specific ethnic subgroups and conducting comparative studies based on ethnicity. Our study focused on the difference between refugees and non-refugees, thus providing an alternative approach to conducting subgroup analysis. It helped to debunk the Asian minority myth that labels Asian immigrants as quiet, hard-working, and successful (Kim \& Kim, 2014).

As for the differences between the two subgroups, refugees often have many traumatic experiences before immigration, cannot return to their home country at will, are expected to adapt to a new culture, and feel guilty about abandoning their homeland and culture. Eisenbruch (1991) has coined a term "cultural bereavement" to describe these experiences. As a result, Southeast Asian refugees in the United States may experience more acculturative stress and are less acculturated than non-refugee immigrants (Dow, 2011). Because they are forced to abandon their culture and homeland, they are more likely to be marginalized in the host society and may become isolated in their private world or start showing antisocial behaviors and mental health issues (Eisenbruch, 1991).

Family factors seem to affect adolescents' psychological well-being similarly in the refugee and non-refugee populations. This is especially the case when it comes to the effect of parent-child conflict. As reviewed earlier, researchers have consistently reported a negatively association between conflict and self-esteem and a positive association between conflict and depressive symptoms across different Asian ethnic groups (e.g., Lui, 2015). However, we speculated that parent-child conflict may be more intense in Southeast Asian refugee families given that refugee parents could experience cultural bereavement as described by Eisenbruch (1991).

\subsection{Hypotheses}

The authors wanted to investigate whether acculturation would directly predict self-esteem and depressive symptoms (Hypothesis 1) and whether acculturation would affect self-esteem and depressive symptoms through parent-child conflict and family cohesion among children of Asian immigrants (Hypothesis 2). We did not specify a direction for the relationship between acculturation and psychological well-being given the mixed findings in the literature. Hypothesis 2 can be further specified as: adolescent acculturation would be associated with higher level of parent-child conflict and lower level of family cohesion, which in turn would be associated with lower self-esteem and more depressive symptoms. In order to compare the differences and similarities between refugee and non-refugee Asian adolescents, we conducted separate analyses for each group.

\section{Methodology}

\subsection{Data and Sample}

In this study, we analyzed the data from the Children of Immigrants Longitudinal Study (CILS; Portes \& Rumbaut, 2001). Tracking children of immigrants for over 10 years, the CILS conducted three separate surveys. We only included data from the follow-up survey because of the lack of relevant data in the original and the third survey. The original survey was conducted in 1992 in San Diego, California and Miami/Ft. Lauderdale, Florida. Participants were recruited in all local middle schools or junior high schools. Students in these schools were screened based on their immigration background. To participate in the original survey, students must be American-born and have at least one foreign-born parent, or foreign-born and have lived in the United States for at least 5 years (Portes \& Rumbaut, 2001). Three years after the original survey, when students were about to graduate from high school, a follow-up survey was conducted and a total of 4,288 students ( $82 \%$ of the original sample) were retrieved and invited to participate. For both surveys, data were collected using paper-and-pencil self-administered questionnaire on youth adaption and growth. Questions were regarding children's demographic characteristics, language use, ethnic identities, self-esteem, depressive symptoms, educational outcomes, etc.

The analytic sample of this study consisted of a total of 1,342 adolescents from Asian immigrant families. We included a subsample of 563 Southeast Asian adolescents, in which 52\% were Vietnamese, 24\% were Laotian, $15 \%$ were Cambodian, and $8 \%$ were Hmong. Also included was a subsample of Asian non-refugee adolescents $(\mathrm{N}=779)$, in which $91 \%$ were Filipino and $9 \%$ were Chinese. 


\subsection{Measures}

\subsubsection{Self-esteem}

Adolescents' self-esteem was measured by the Rosenberg Self-Esteem Scale (Rosenberg, 1965). It consisted of 10 items rated on a four-point scale (e.g., "I take a positive attitude toward myself."). Responses ranged from $0=$ "disagree a lot" to 3 "agree a lot". Negative items were recoded and a total score was calculated to indicate overall self-esteem (with a range from 0 to 30 ). In the study sample, the reliability score for the scale was .81 .

\subsubsection{Depressive Symptoms}

Depressive symptoms were assessed using a shortened version of the Center for Epidemiological Studies-Depression Scale (Radloff, 1977). This version of the scale included a total of four items which were representative of the original scale and proved good enough for the use among children of immigrants (Rumbaut \& Portes, 2001). One sample item was "I felt depressed". All questions were scored on a four-point Likert-type scale ranging from " $1=$ rarely" to " $4=$ most of the time". A mean score of all was calculated to indicate the overall level of depressive symptoms (with a range from 1 to 4$)$. In the sample, the reliability score for this scale was .75.

\subsubsection{Acculturation}

Three proxy measures on acculturation were included as independent variables: nativity, time in the United States, and U.S. preference. Nativity was coded as a categorical variable (U.S.-born $=0$; foreign-born $=1$ ). We deem foreign-born adolescents to be less acculturated than the U.S.-born adolescents, as done in previous research (e.g., Ebin et al., 2001). Time in the United States was assessed by asking "How long have you lived in the U.S.?" Responses were reverse coded as follows: less than five years $=1$, five to nine years $=2$, ten years or more $=3$, and all my life $=4$. Thus, respondents who reported a higher score were more acculturated. Finally, U.S. preference was measured by asking "How often do you prefer American ways of doing things?" Responses were reverse coded so that a higher score indicates a higher level of acculturation (i.e., never $=1$, sometimes $=2$, most of the time $=3$, and all of the time $=4$ ).

\subsubsection{Parent-Child Conflict}

Adolescents' perceptions of parent-child conflict were assessed by the parent-child conflict index. Including four items, the index was identified through factor analyses and has been used in previous research (Portes \& Rumbaut, 2001; Ying $\&$ Han, 2007). An exemplary item was "How often do you get in trouble because your way of doing things is different from that of your parents?". Responses were based on a four-point scale, ranging from "all of the time" or "very true" to "never" or "not true at all." Items were recoded so that higher scores indicate higher levels of conflict. The reliability score was equal to .72 in the sample.

\subsubsection{Family Cohesion}

Family cohesion was measured by the family cohesion index which consisted of three items. The index was identified through factor analyses and has been used in previous research (Portes \& Rumbaut, 2001). Each item was rated on a five-point Likert-type scale, ranging from "never" to "always". An exemplary item was "Family members like to spend free time with each other". The mean of the three items was calculated to show the overall level of family cohesion (with a range of 1 to 5). This index had a reliability score of .82 .

\subsection{Analysis Strategy}

Data analysis was conducted with Stata 13.0 and Mplus 7.4. We conducted bivariate analyses to explore the data, which was followed by structural equation modeling (SEM) path analyses. Acculturation was not modeled as a latent variable because mixing dichotomous and continuous indicators for a latent variable is difficult to interpret (Bray, Smith, Piper, Roberts, \& Baker, 2016). We employed commonly used symbols (i.e., $a, b, c$, and $c^{\prime}$ ) to denote the paths between the independent variables, dependent variables, and the mediator variables. Path $a$ represents the effect of acculturation on the proposed mediator, whereas path $b$ is the effect of the mediator on the psychological well-being controlling for the effect of acculturation. Path $c$ (without the mediator in regression) and path $c^{\prime}$ (with the mediator in regression) are the total and the direct effect of acculturation on the psychological well-being, respectively. Finally, sex as a control variable was included in all path models.

To test the statistical significance of the indirect effect, we used the bootstrap method with 5,000 iterations in Mplus. Bias-corrected confidence intervals (BC-CI) are reported to determine whether the indirect effect is significant. To compare the difference and similarities between the refugee and non-refugee subgroups, we conducted a multiple group SEM analysis in Mplus. We reported the goodness of fit using indices such as the chi square test $\left(\chi^{2}\right)$, the Comparative Fit Index (CFI), the Tucker-Lewis Index (TLI), and the Root Mean Square Error of Approximation (RMSEA). Finally, the $\chi^{2}$ difference test was performed to determine better fit for alternative models. 


\section{Results}

\subsection{Descriptive Statistics}

Table 1 shows the differences in the study variables between the refugee group and the non-refugee group. Compared to adolescents in refugee families, adolescents in non-refugee families were more likely to be U.S.-born $\left(\chi^{2}=322.60, p\right.$ $<.001)$, to live longer in the United States $\left(\chi^{2}=248.17, p<.001\right)$, and to prefer American ways of doing things $(t=6.35$, $p<.001)$, These indicated that they were more acculturated than their refugee counterparts. They also had higher self-esteem $(t=5.38, p<.001)$ and experienced lower parent-child conflict $(t=-2.19, p<.01)$ than the refugee group. However, they displayed more depressive symptoms than their refugee counterparts $(t=1.90, p<.05)$. There was no difference in family cohesion or sex between the two groups.

\subsection{Correlations}

Table 2 shows the correlations between all study variables for both refugee adolescents and non-refugee adolescents. For both groups, self-esteem was negatively correlated with parent-child conflict and positively with family cohesion; depressive symptoms were positively correlated with parent-child conflict and negatively with family cohesion. Parent-child conflict and family cohesion were negatively correlated. Self-esteem and depressive symptoms were also negatively correlated. In addition, self-esteem was significantly correlated with nativity among non-refugee adolescents, but not among refugee adolescents. Time in the United States was positively correlated with self-esteem among refugee adolescents but not non-refugee adolescents.

\subsection{Test of Model Fit}

The baseline model (Model 1) was an unconstrained model in which all regression weights and covariances were free to be estimated for both the refugee and non-refugee subsample. Results indicated that the model fit the data well $\left(\chi^{2}=\right.$ 9.69, $\mathrm{df}=6, p=.14 ; \mathrm{CFI}=.99 ; \mathrm{TLI}=.97 ; \mathrm{RMSEA}=.03$ ).

Model 2 included constraints on the regression weights for all significant $a, b$, and $c^{\prime}$ paths based on the results of Model 1. These paths may be statistically significant in the non-refugee group, the refugee group, or both groups. In Model 2, all significant weights were set equal between the refugee and the non-refugee groups. Fit indices indicated a worse fit of Model 2 than Model 1: $\chi^{2}=42.84, \mathrm{df}=19, p<.01 ; \mathrm{CFI}=.97$; TLI $=.94$; RMSEA $=.04$. Furthermore, a $\chi^{2}$ difference test between Model 1 and Model 2 supported the better fit of the baseline model $\left(\mathrm{D}_{\chi^{2}}=33.15, \mathrm{D}_{\mathrm{df}}=13, p\right.$ $=.002$ ). Therefore, Model 1 in which all regression paths for both groups were freely estimated was retained.

Table 1. Descriptive statistics on all study variables by immigration type

\begin{tabular}{|c|c|c|c|c|}
\hline Variable & $\begin{array}{l}\text { Total sample } \\
(n=1,342)\end{array}$ & $\begin{array}{l}\text { Refugees } \\
(n=563) \\
\end{array}$ & $\begin{array}{l}\text { Non-Refugees } \\
(n=779)\end{array}$ & $t / \chi^{2}$ \\
\hline & $M(S D)$ or $\%$ & $M(S D)$ or $\%$ & $M(S D)$ or $\%$ & \\
\hline Self-esteem & $22.61(5.20)$ & $21.73(5.15)$ & $23.26(5.14)$ & $5.38 * * *$ \\
\hline Depressive symptoms & $6.77(2.55)$ & $6.62(2.50)$ & $6.89(2.59)$ & $1.90 *$ \\
\hline Parent-adolescent conflict & $1.93(0.67)$ & $1.97(0.67)$ & $1.89(0.67)$ & $-2.19 * *$ \\
\hline Family cohesion & $3.51(0.96)$ & $3.47(0.99)$ & $3.53(0.94)$ & 1.05 \\
\hline Nativity & & & & $322.60 * * *$ \\
\hline Foreign born & 63.11 & 90.94 & 43.00 & \\
\hline U.S. born (Reference group) & 36.89 & 9.06 & 57.00 & \\
\hline Years in the United States & & & & $248.17 * * *$ \\
\hline Less than 10 years & 18.33 & 21.14 & 16.30 & \\
\hline 10 years or more & 48.14 & 68.74 & 33.25 & \\
\hline All my life & 33.53 & 10.12 & 50.45 & \\
\hline U.S. preference & $2.50(0.62)$ & $2.38(0.57)$ & $2.59(0.63)$ & $6.35 * * *$ \\
\hline Sex & & & & 0.28 \\
\hline Female adolescents & 50.30 & 51.15 & 49.68 & \\
\hline Male adolescents & 49.70 & 48.85 & 50.32 & \\
\hline
\end{tabular}

\subsection{Total Effects}

For the immigrant group, nativity and time in the United Sates predicted self-esteem significantly $(B=.14, S E=.06, p$ $=.01 ; B=.16, S E=.06, p<.01$; respectively); however, U.S. preference was not a significant predictor for self-esteem. All three acculturation measures were not significantly associated with depressive symptoms. For the refugee group, time in the United States predicted self-esteem significantly $(B=.22, S E=.05, p<.001)$. Similar to the immigrant group, none of the three measures was associated with depressive symptoms. 
Table 2. Zero-order correlation coefficients among research variables by immigration type

\begin{tabular}{|c|c|c|c|c|c|c|c|}
\hline Variable & 1 & 2 & 3 & 4 & 5 & 6 & 7 \\
\hline 1. Self-esteem & - & $-.39 * * *$ & $-.34 * * *$ & $.18^{* * *}$ & -.03 & $.15 * * *$ & .06 \\
\hline 2. Depressive symptoms & $-.45 * * *$ & - & $.36 * * *$ & $-.21 * * *$ & .03 & -.06 & -.01 \\
\hline 3. Parent-adolescent conflict & $-.35 * * *$ & $.31 * * *$ & - & $-.39 * * *$ & -.01 & $.10^{*}$ & $.09^{*}$ \\
\hline 4. Family cohesion & $.26 * * *$ & $-.20 * * *$ & $-.36 * * *$ & - & .05 & $-.18 * * *$ & -.07 \\
\hline 5. Nativity & .02 & .02 & $.09^{*}$ & .02 & - & $-.64 * * *$ & $-.11 * *$ \\
\hline 6. Years in the U.S. & .04 & -.03 & $-.08 *$ & -.06 & $-.78 * * *$ & - & $.14 * * *$ \\
\hline 7. U.S. preference & .02 & -.02 & -.01 & -.05 & $-.12 * * *$ & $.15 * * *$ & - \\
\hline 8. Sex & $-.10 * *$ & $.20 * * *$ & $.09 * *$ & -.05 & -.02 & .05 & .03 \\
\hline
\end{tabular}

Note. Upper triangle is refugees; Lower triangle is non-refugees.

\subsection{Direct Effects}

Figure 1 and Figure 2 contain the standardized estimate and its significance level of all the direct paths in Model 1 for the refugee group and non-refugee group, respectively. Comparing with total effects of acculturation on psychological well-being, more direct paths were significant in the two groups. Specifically, time in the United States was positively related to self-esteem in both groups (refugee: $B=.29, S E=.05, p<.001$; non-refugee: $B=.16, S E=.06, p<.01$ ). Foreign-born adolescents reported higher self-esteem than U.S.-born adolescents in both groups (refugee: $B=.15, S E$ $=.06, p<.01$; non-refugee: $B=.17, S E=.05, p<.01)$. U.S. preference was not associated with self-esteem or depressive symptoms for either group. As for the difference between the two groups, time in the United States negatively predicted depressive symptoms in the refugee group $(B=-.14, S E=.05, p<.01)$; however, there was no direct association between the two variables in the non-refugee group.

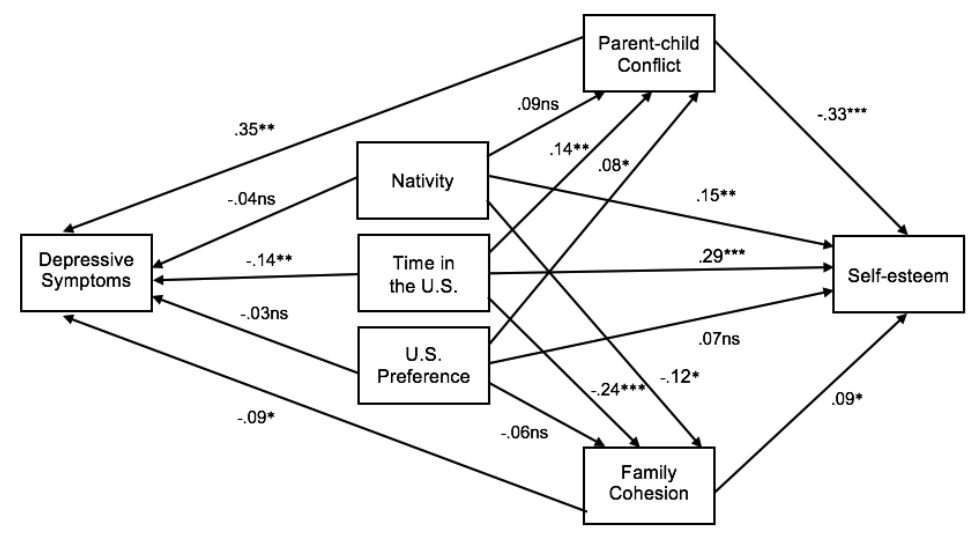

Figure 1. Standardized coefficients and $p$ values of the path models of the relationships between acculturation and self-esteem and depressive symptoms as mediated by parent-child conflict and family cohesion among adolescents from Southeast Asian refugee families

Note. ns=not significant. ${ }^{*} \mathrm{p}<.05 .{ }^{* *} \mathrm{p}<.01 . * * * \mathrm{p}<.001$. Results of gender omitted in this figure for simplicity.

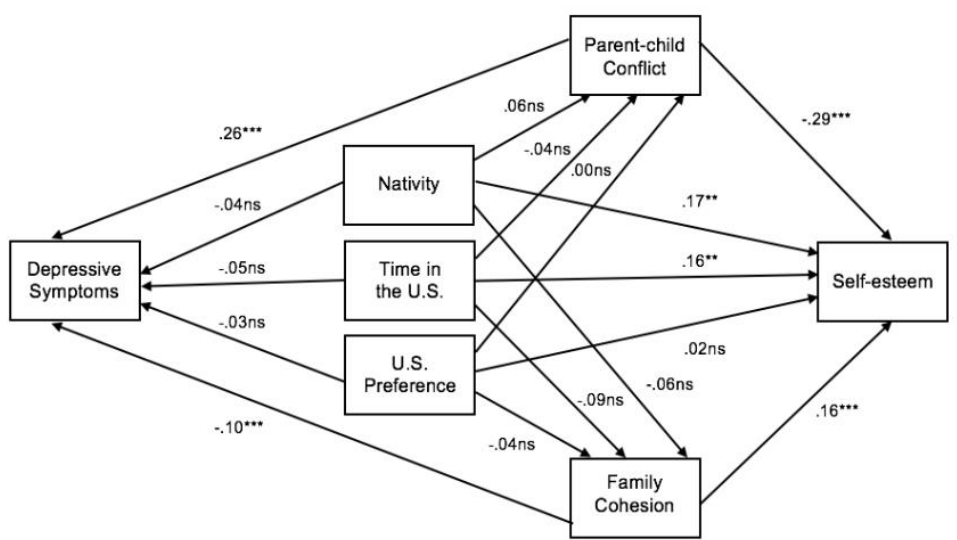

Figure 2. Standardized coefficients and $p$ values of the path models of the relationships between acculturation and self-esteem and depressive symptoms as mediated by parent-child conflict and family cohesion among adolescents from non-refugee Asian families

Note. ns=not significant. ${ }^{*} \mathrm{p}<.05 .{ }^{*} \mathrm{p}<.01 . * * * \mathrm{p}<.001$. Results of gender omitted in this figure for simplicity. 


\subsection{Indirect Effects}

Table 3 shows the results of the indirect effects of acculturation on psychological well-being through family dynamics among adolescents from refugee families (Results for non-refugee families were omitted due to lack of significant indirect effects in this group). Standardized estimates, standard errors, and the 95\% BC-CI for the indirect effects are reported. If a confidence interval does not contain 0 , then the corresponding indirect effect is considered statistically significant.

As shown in Table 3, the following eight indirect paths were significant in the refugee subsample: family cohesion mediated the associations between nativity and self-esteem $(\mathrm{BC}-\mathrm{CI}=[-.03,-.001])$ and depressive symptoms $(\mathrm{BC}-\mathrm{CI}=$ $[.00, .03])$; parent-child conflict mediated the associations between time in the United States and self-esteem (BC-CI = $[-.09,-.01])$ and depressive symptoms $(\mathrm{BC}-\mathrm{CI}=[.01, .09])$; family cohesion mediated the associations between time in the United States and self-esteem $(\mathrm{BC}-\mathrm{CI}=[-.05,-.00])$ and depressive symptoms $(\mathrm{BC}-\mathrm{CI}=[.002, .05])$; parent-child conflict mediated the associations between U.S. preference and self-esteem $(\mathrm{BC}-\mathrm{CI}=[-.06,-.001])$ and depressive symptoms $(\mathrm{BC}-\mathrm{CI}=[.001, .06])$. These findings supported hypothesis 2 in the refugee group.

However, in the refugee group, parent-child conflict did not mediate between nativity and self-esteem or depressive symptoms; family cohesion did not mediate between U.S. preference and self-esteem or depressive symptoms. Nevertheless, as shown in Figure 1, parent-child conflict was negatively related to self-esteem $(B=-.33, S E=.04, p$ $<.001)$, but positively with depressive symptoms $(B=.35, S E=.05, p<.001)$; family cohesion was positively related to self-esteem $(B=.09, S E=.05, p<.05)$, but negatively with depressive symptoms $(B=-.09, S E=.05, p<.05)$.

None of the 12 indirect paths from acculturation to psychological well-being via family dynamics was statistically significant in the non-refugee subsample thus providing no evidence for hypothesis 2. Nevertheless, as shown in Figure 2 , parent-child conflict was negatively related to self-esteem $(B=-.29, S E=.04, p<.01)$, but positively with depressive symptoms $(B=.26, S E=.04, p<.001)$; family cohesion was positively related to self-esteem $(B=.16, S E=.04, p$ $<.001)$, but negatively with depressive symptoms $(B=-.10, S E=.04, p=.01)$.

Table 3. Results of the Indirect Effects of Acculturation on Psychological Well-Being through Family Dynamics among Adolescents from Asian Refugee Families

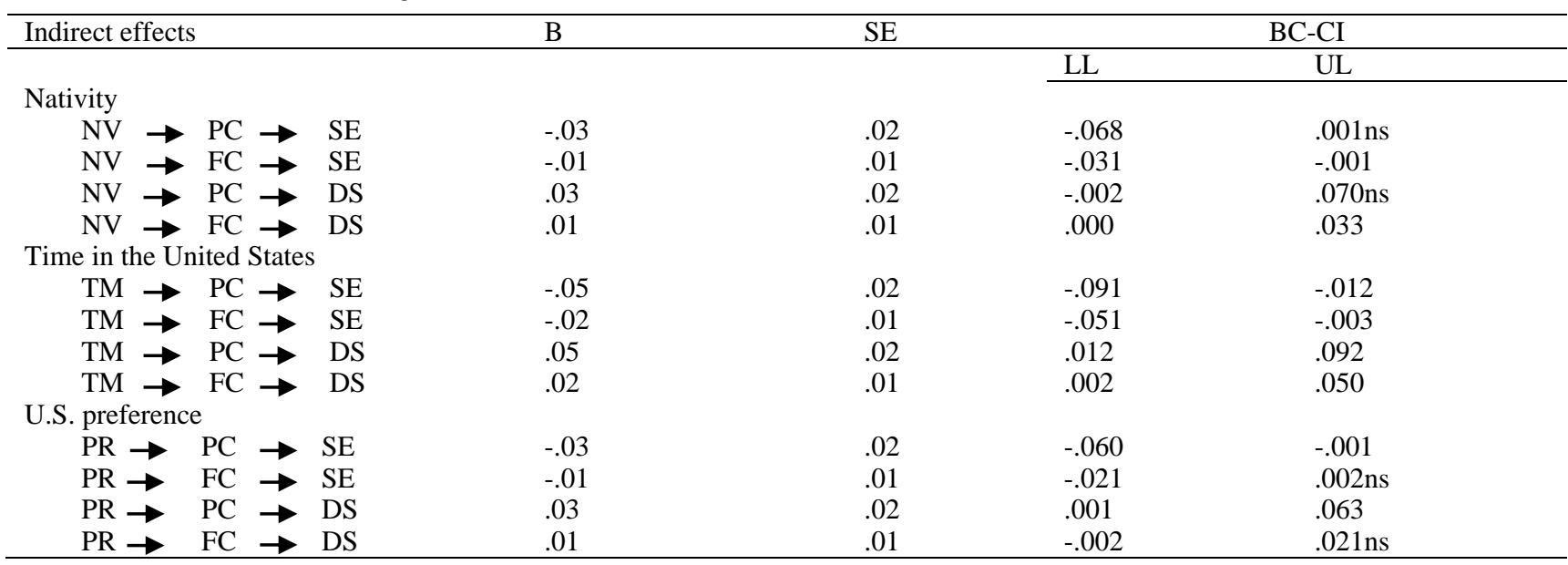

Note. $\mathrm{NV}=$ nativity. $\mathrm{TM}=$ time in the United States. $\mathrm{PR}=$ U.S. preference. $\mathrm{PC}=$ Parent-child conflict. $\mathrm{FC}=$ family cohesion. $\mathrm{SE}=$ self-esteem. $\mathrm{DS}=$ depressive symptoms. $\mathrm{B}=$ standardized coefficients. $\mathrm{SE}=$ bootstrap standard errors. $\mathrm{BC}-\mathrm{CI}=$ bias-corrected confidence intervals, $\mathrm{LL}=$ lower limit, $\mathrm{UL}=$ upper limit. $\mathrm{ns}=$ not significant.

\section{Discussion}

This study examined the direct and indirect effects of acculturation on psychological well-being among Asian children of immigrants. Consistent with Hypothesis 1, our findings suggest that acculturation was generally associated with psychological well-being, although one of the three measures of acculturation (i.e., U.S. preference) was not associated with psychological-being. The nature of the relationship depended on the measure of acculturation and the type of migration. For instance, measured by time in the United States, higher level of acculturation of adolescents in both groups (i.e., longer time in the United States) was related to higher self-esteem. On the other hand, lower level of acculturation measured by nativity (i.e., adolescents being foreign-born) in both groups was associated with higher level self-esteem. In terms of subgroup differences, time in the United States was negatively associated with depressive symptoms in the refugee group, but not in the immigrant group.

Previous research is inconclusive about the effect of acculturation on the adolescent psychological well-being. For 
instance, more acculturated Asian-American adolescents of non-refugee origin have reported fewer depressive symptoms (Crane, Ngai, Larson, \& Hafen, 2005); however, an ethnically diverse group of Asian adolescents (including refugee and non-refugee origin) reported lower self-esteem when they became more acculturated (Rhee, Chang, \& Rhee, 2003). To reconcile these conflicting findings, researchers have suggested that the effect of acculturation on health may be contingent on what health outcome or ethnic group was studied (Salant \& Lauderdale, 2003). Based on our study, it appears that measures of acculturation also make a difference.

More importantly, we found eight out of the 12 indirect paths to be significant among adolescents from refugee families but not non-refugee families, thus Hypothesis 2 was partially supported. Generally speaking (with the exception of the indirect relationship from nativity to psychological well-being through family cohesion), more acculturated Asian refugee adolescents experienced lower family cohesion and higher parent-child conflict, which in turn were related to more psychological problems such as lower self-esteem and more depressive symptoms. In other words, acculturation seems to put the adolescents from refugee families at higher risk by heightening parent-child conflict and weakening family cohesion. These findings highlight the importance of examining the indirect effects of acculturation on the psychological well-being through family dynamics among refugee adolescents. In this direction, more studies are needed that examine the effect of acculturation in family, school and societal contexts (e.g., Schwartz, Unger, Zamboanga, \& Szapocznik, 2010).

The differential findings across the two groups could be attributed to the cultural bereavement phenomenon experienced by refugee parents. According to Eisenbruch (1991), refugees were faced with fewer resources and heightened psychosocial stress in the new county. These, in turn, could predict higher parent-child conflict in refugee families and more psychological problems in children from these families.

Meanwhile, researchers have proposed and tested the acculturation gap hypothesis (for a review, see Telzer, 2011). Acculturation gap (i.e., parent-child discrepancy in acculturation) has been found to be associated with higher psychological distress and greater risk for clinical depression among Asian college students (Hwang \& Wood, 2009) and high school adolescents of Chinese origin (Hwang, Wood, \& Fujimoto, 2010). We believe that the acculturation gap could be even more pronounced in refugee families. Refugee parents may have more difficulties in learning English, seeking employment, fighting the lingering effects of premigration trauma, and reestablishing social support after immigration, all of which have been linked to lower acculturation levels (Organista, Organista, \& Kurasaki, 2003). Refugee parents' lower acculturation level, coupled with their children's relatively higher acculturation level, contributes to the acculturation gap between the two generations. As a result, children of Asian refugees may experience higher parent-child conflict and lower family cohesion, which in turn are linked to psychological problems. Previous research supported an indirect relationship between acculturation discrepancies and more depressive symptoms through heightened parent-child conflict among children from southeast Asian refugee families (Ying \& Han, 2007). Our findings complemented Ying and Han's (2007) study because we discovered the indirect effects of acculturation on psychological well-being through family cohesion.

Although only eight out of 12 mediational paths were statistically significant for the refugee subgroup in our study, it is worth noting that, across both groups, parent-child conflict was a risk factor and family cohesion was a protective factor for the psychological well-being (see Figure $1 \& 2$ ). These findings are consistent with many previous studies (e.g., Juang \& Alvarez, 2010; Lui, 2015). However, being foreign born predicted lower family cohesion in the refugee subsample $(B=-.12, S E=.05, p<.05$; see Figure 1$)$. This could have to do with using nativity as a proxy measure for acculturation in our study. All foreign-born adolescents in our sample were brought to the United States at an early age (by age 12), which may help to bridge the difference in acculturation between foreign-born and U.S.-born adolescents from refugee families.

\subsection{Limitations and Directions for Future Research}

Just as any other study, this study has some limitations. First, acculturation was assessed by proxies rather than standardized questionnaires. The use of proxy measures could be problematic because acculturation is understood as a linear process from non-acculturated to fully acculturated; however, researchers continue using such measures in acculturation research (Koneru, de Mamani, Flynn, \& Betancourt, 2007). Nevertheless, future studies need to tap into the role of multidimensional acculturation. One such instrument is the Bicultural Involvement Questionnaire (BIQ). It measures the involvement in both the ethnic culture and the American mainstream culture by including questions on language, food, recreation, and media use (Szapocznik \& Kurtines, 1980). Evidence has increasingly suggested the protective role of biculturalism in shaping adolescent psychological outcomes (e.g., Smokowski \& Bacallao, 2007; Smokowski, Rose, \& Bacallao, 2008), thus using a multidimensional measure of acculturation will probably allow researchers to reconcile the conflicting evidence on the association between acculturation and psychological well-being.

In addition, our study proposed rather simple mechanisms through which acculturation affects psychological well-being 
of the children from refugee families. Whenever possible, future researchers need to take a mixed methods approach to studying the underlying mechanisms. For example, they may conduct in-depth interviews with refugee parents and their children to understand the complicated process of acculturation of both generations and investigate how it may impact psychological well-being of the children. Meanwhile, considering foreign-born refugee adolescents reported lower family cohesion and higher self-esteem in our study, we suggest studying how foreign-born and native-born children of refugees perceive family cohesion differently. Quantitatively, future researchers may want to test the acculturation discrepancy hypothesis using samples of refugee parents and their children (Telzer, 2011).

Finally, our study was based on southeast Asian immigrants who entered the United States in the late 1970s and early 1980s. Because the CILS Data were collected in the mid- and late-1990s, the age and relevance of the data are somewhat questionable. Nevertheless, the CILS is by far the largest dataset on children of immigrants. When conducting a replication study on a large scale was not feasible, it afforded us an opportunity to conduct an Asian subgroup analysis on the relationship between acculturation and psychological well-being. Nevertheless, as the second-generation children of immigrants grew up and had their own children, future researchers could build on the CILS and investigate the well-being of the third-generation children of immigrants. Alternatively, researchers can replicate the CILS using more recent refugee populations from other parts of Asia, including Syria, Burma, and Iraq.

\subsection{Practice Implications}

Given the complicated role of acculturation in shaping psychological well-being of adolescents from Asian immigrant families, professionals who work with Asian immigrant families must include acculturation in the assessment of their clients to provide culturally competent services (Kohli, Huber, \& Faul, 2010). In doing so, they should pay extra attention to immigrant clients' cultural values and behaviors that may be incongruent with American mainstream cultural values and behaviors (Berte, 2015).

In addition, professionals who work with Asian refugee families need to develop and implement family interventions that take the roles of family cohesion parent-child conflict into account. By incorporating program elements that promote family cohesion and deescalate parent-child conflict, they may be in a better position to improve adolescent psychological well-being. For example, a psychoeducational class that teaches Asian immigrant parents the difference between their ethnic cultures and the U.S. mainstream culture will be useful. Parent-child differences in acculturation and intercultural and/or intergenerational conflict may be minimized. This will likely lead to better psychological well-being among adolescents from Asian immigrant families. This type of intervention has been designed and tested among immigrant families. One example is the Strengthening Intergenerational/Inter-cultural Ties in Immigrant Families program (SITIF). Although evidence has shown the SITIF was effective in reducing parent-child conflict in immigrant Chinese families (Ying, 2009), efforts to implement it in other immigrant families, especially the Southeast Asian refugee families, are warranted.

\section{Conclusion}

In conclusion, our study provided partial evidence that higher level acculturation (measured by longer time in the United States and higher level of U.S. preference) is related to higher parent-child conflict and lower family cohesion, which in turn are related to lower psychological well-being (i.e., lower self-esteem and more depressive symptoms) of adolescents in Asian refugee families. These findings may be important in a number of ways: First, they challenged the popular perception of Asian immigrants being a "model minority". Given the differences between children in Southeast Asian refugee families and Asian non-refugee families, professionals who work with these families may need to pay more attention to the refugee population in practice and research. Second, our study validated the importance for acculturation studies to incorporate a broader social ecological framework that considers the role of environmental factors such as parent-child conflict, social networks, and public policy (Schwartz et al., 2010). Finally, since parent-child conflict and family cohesion appear to play a more immediate and important role in shaping psychological well-being among adolescents in refugee families, professionals who work with refugee families need to incorporate these factors in program development and implementation.

\section{References}

Baer, J. C., \& Schmitz, M. F. (2007). Ethnic differences in trajectories of family cohesion for Mexican American and non-Hispanic white adolescents. Journal of Youth and Adolescence, 36, 583-592. https://doi.org/10.1007/s10964-007-9177-3

Berry, J. W. (2003). Conceptual approaches to acculturation. In K. M. Chun, P. B. Organista \& G. Martin (Eds.), Acculturation: Advances in theory, measurement, and applied research (pp. 17-38). Washington, DC: American Psychological Association. https://doi.org/10.1037/10472-004

Berte, D. Z. (2015). Mental health issues in immigrant communities. In F. Chang-Muy \& E. P. Congress (Eds.), Social 
work with immigrants and refugees: Legal issues, clinical skills, and advocacy (2nd ed., pp. 143-174). New York, NY: Springer Publishing Company.

Birman, D., \& Taylor-Ritzler, T. (2007). Acculturation and psychological distress among adolescent immigrants from the former Soviet Union: Exploring the mediating effect of family relationships. Cultural Diversity and Ethnic Minority Psychology, 13, 337-346. https://doi.org/10.1037/1099-9809.13.4.337

Bray, B. C., Smith, R. A., Piper, M. E., Roberts, L. J., \& Baker, T. B. (2016). Transitions in smokers' social networks after quit attempts: A latent transition analysis. Nicotine \& Tobacco Research, 18, 2243-2251. https://doi.org/10.1093/ntr/ntw173

Cheng, H. L., Lin, S. P., \& Cha, C. H. (2015). Perceived discrimination, intergenerational family conflicts, and depressive symptoms in foreign-born and U.S.-born Asian American emerging adults. Asian American Journal of Psychology, 6, 107-116. https://doi.org/10.1037/a0038710

Choi, Y., He, M., \& Harachi, T. W. (2008). Intergenerational cultural dissonance, parent-child conflict and bonding, and youth problem behaviors among Vietnamese and Cambodian immigrant families. Journal of Youth and Adolescence, 37, 85-96. https://doi.org/10.1007/s10964-007-9217-z

Crane, D. R., Ngai, S. W., Larson, J. H., \& Hafen, M. (2005). The influence of family functioning and parent-adolescent acculturation on North American Chinese adolescent outcomes. Family Relations, 54, 400-410. https://doi.org/10.1111/j.1741-3729.2005.00326.x

De La Rosa, M. (2002). Acculturation and Latino adolescents' substance use: A research agenda for the future. Substance Use \& Misuse, 37, 429-456. https://doi.org/10.1081/JA-120002804

Diego, M. A., Field, T. M., \& Sanders, C. E. (2003). Academic performance, popularity, and depression predict adolescent substance use. Adolescence, 38, 35-42.

Dow, H. D. (2011). The acculturation processes: The strategies and factors affecting the degree of acculturation. Home Health Care Management \& Practice, 23, 221-227. https://doi.org/10.1177/1084822310390877

Ebin, V. J., Sneed, C. D., Morisky, D. E., Rotheram-Borus, M. J., Magnusson, A. M., \& Malotte, C. K. (2001). Acculturation and interrelationships between problem and health-promoting behaviors among Latino adolescents. Journal of Adolescent Health, 28, 62-72.

Eisenbruch, M. (1991). From post-traumatic stress disorder to cultural bereavement: Diagnosis of Southeast Asian refugees. Social Science \& Medicine, 33, 673-680. https://doi.org/10.1016/0277-9536(91)90021-4

Gordon, M. M. (1964). Assimilation in American life: The role of race, religion, and national origins. New York: Oxford University Press.

Han, M., \& Lee, M. (2011). Risk and protective factors contributing to depressive symptoms in Vietnamese American college students. Journal of College Student Development, 52, 154-166. https://doi.org/10.1353/csd.2011.0032

Huang, K. Y., Calzada, E., Cheng, S., \& Brotman, L. M. (2012). Physical and mental health disparities among young children of Asian immigrants. The Journal of pediatrics, 160, 331-336. https://doi.org/10.1016/j.jpeds.2011.08.005

Hwang, W. C., \& Ting, J. Y. (2008). Disaggregating the effects of acculturation and acculturative stress on the mental health of Asian Americans. Cultural Diversity and Ethnic Minority Psychology, 14, 147. https://doi.org/10.1037/1099-9809.14.2.147

Hwang, W. C., \& Wood, J. J. (2009). Acculturative family distancing: Links with self-reported symptomatology among Asian Americans and Latinos. Child Psychiatry and Human Development, 40, 123-138. https://doi.org/10.1007/s10578-008-0115-8

Hwang, W. C., Wood, J. J., \& Fujimoto, K. (2010). Acculturative family distancing (AFD) and depression in Chinese American families. Journal of Consulting and Clinical Psychology, 78, 655-667. https://doi.org/10.1037/a0020542

Isomaa, R., Väänänen, J. M., Fröjd, S., Kaltiala-Heino, R., \& Marttunen, M. (2013). How low is low? Low self-esteem as an indicator of internalizing psychopathology in adolescence. Health Education \& Behavior, 40, 392-399. https://doi.org/10.1177/1090198112445481

Juang, L. P., \& Alvarez, A. A. (2010). Discrimination and adjustment among Chinese American adolescents: Family conflict and family cohesion as vulnerability and protective factors. American Journal of Public Health, 100, 2403-2409. https://doi.org/10.2105/AJPH.2009.185959

Kalibatseva, Z., \& Leong, F. T. (2011). Depression among Asian Americans: Review and recommendations. Depression Research and Treatment, 2011, 1-9. https://doi.org/10.1155/2011/320902 
Kiang, L., Tseng, V., \& Yip, T. (2016). Placing Asian American child development within historical context. Child Development, 87, 995-1013. https://doi.org/10.1111/cdev.12578

Kim, B. S., Yang, P. H., Atkinson, D. R., Wolfe, M. M., \& Hong, S. (2001). Cultural value similarities and differences among Asian American ethnic groups. Cultural Diversity and Ethnic Minority Psychology, 7, 343. https://doi.org/10.1037/1099-9809.7.4.343

Kim, I., \& Kim, W. (2014). Post-resettlement challenges and mental health of Southeast Asian Refugees in the United States. Best Practices in Mental Health, 10, 63-77.

Kohli, H. K., Huber, R., \& Faul, A. C. (2010). Historical and theoretical development of culturally competent social work practice. Journal of Teaching in Social Work, 30, 252-271. https://doi.org/10.1080/08841233.2010.499091

Koneru, V. K., de Mamani, A. G. W., Flynn, P. M., \& Betancourt, H. (2007). Acculturation and mental health: Current findings and recommendations for future research. Applied and Preventive Psychology, 12, 76-96. https://doi.org/10.1016/j.appsy.2007.07.016

Lara, M., Gamboa, C., Kahramanian, M. I., Morales, L. S., \& Bautista, D. E. (2005). Acculturation and Latino health in the United States: A review of the literature and its sociopolitical context. Annual Review of Public Health, 26, 367-397. https://doi.org/10.1146/annurev.publhealth.26.021304.144615

Le, M. N., \& Nguyen, T. U. (2012). Social and cultural influences on the health of the Vietnamese American population. In G. J. Yoo, M.-N. Le, \& A. Y. Oda (Eds.), Handbook of Asian American health (pp. 87-101). New York, NY: Springer Science \& Business Media.

Leong, F., Park, Y. S., \& Kalibatseva, Z. (2013). Disentangling immigrant status in mental health: Psychological protective and risk factors among Latino and Asian American immigrants. American Journal of Orthopsychiatry, 83, 361-371. https://doi.org/10.1111/ajop.12020

Li, Y. (2013). Intergenerational Conflict, Attitudinal Familism, and Depressive Symptoms Among Asian and Hispanic Adolescents in Immigrant Families: A Latent Variable Interaction Analysis. Journal of Social Service Research, 40, 80-96. https://doi.org/10.1080/01488376.2013.845128

Li, Y., \& Warner, L. A. (2015). Parent-adolescent conflict, family cohesion and self-esteem among Hispanic adolescents in immigrant families: A comparative analysis. Family Relations, 64, 579-591. https://doi.org/10.1111/fare.12158

Lui, P. P. (2015). Intergenerational cultural conflict, mental health, and educational outcomes among Asian and Latino/a Americans: Qualitative and meta-analytic review. Psychological Bulletin, 141, 404-446. https://doi.org/10.1037/a0038449

Marsiglia, F. F., Parsai, M., \& Kulis, S. (2009). Effects of familism and family cohesion on problem behaviors among adolescents in Mexican immigrant families in the southwest U.S. Journal of Ethnic \& Cultural Diversity in Social Work, 18, 203-220. https://doi.org/10.1080/15313200903070965

Ngo, B., \& Lee, S. J. (2007). Complicating the image of model minority success: A review of Southeast Asian American education. Review of Educational Research, 77, 415-453. https://doi.org/10.3102/0034654307309918

Organista, P. B., Organista, K. C., \& Kurasaki, K. (2003). The relationship between acculturation and ethnic minority mental health. In K. M. Chun, P. B. Organista \& G. Marin (Eds.), Acculturation: Advances in theory, measurement, and applied research (pp. 139-161). Washington, DC: American Psychological Association. https://doi.org/10.1037/10472-010

Portes, A., \& Rumbaut, R. G. (2001). Legacies: The story of the immigrant second generation. Berkeley, CA: University of California Press.

Radloff, L. S. (1977). The CES-D Scale: A self report depression scale for research in the general population. Applied Psychological Measurement, 1, 385-401. https://doi.org/10.1177/014662167700100306

Rhee, S., Chang, J., \& Rhee, J. (2003). Acculturation, communication patterns, and self-esteem among Asian and Caucasian American adolescents. Adolescence, 38, 749-768.

Rosario-Sim, M. G., \& O’Connell, K. A. (2009). Depression and language acculturation correlate with smoking among older Asian American adolescents in New York City. Public Health Nursing, 26, 532-542. https://doi.org/10.1111/j.1525-1446.2009.00811.x

Rosenberg, M. (1965). Society and the adolescent self-image. Princeton, N.J.: Princeton University Press. https://doi.org/10.1515/9781400876136

Rumbaut, R. G., \& Portes, A. (2001). Ethnicities: Children of immigrants in America. Berkeley, CA: University of 
California Press.

Salant, T., \& Lauderdale, D. S. (2003). Measuring culture: A critical review of acculturation and health in Asian immigrant populations. Social Science \& Medicine, 57, 71-90. https://doi.org/10.1016/S0277-9536(02)00300-3

Schwartz, S. J., Unger, J. B., Zamboanga, B. L., \& Szapocznik, J. (2010). Rethinking the concept of acculturation: Implications for theory and research. American Psychologist, 65, 237-251. https://doi.org/10.1037/a0019330

Smokowski, P. R., \& Bacallao, M. L. (2007). Acculturation, internalizing mental health symptoms, and self-esteem: Cultural experiences of Latino adolescents in north Carolina. Child Psychiatry \& Human Development, 37, 273-292. https://doi.org/10.1007/s10578-006-0035-4

Smokowski, P. R., Rose, R., \& Bacallao, M. L. (2008). Acculturation and Latino family processes: How cultural involvement, biculturalism, and acculturation gaps influence family dynamics. Family Relations, 57, 295-308. https://doi.org/10.1111/j.1741-3729.2008.00501.x

Spirito, A., \& Esposito-Smythers, C. (2006). Attempted and completed suicide in adolescence. Annual Review of Clinical Psychology, 2, 237-266. https://doi.org/10.1146/annurev.clinpsy.2.022305.095323

Szapocznik, J., \& Kurtines, W. M. (1980). Acculturation, biculturalism, and adjustment among Cuban Americans. In A. Padilla (Ed.), Acculturation: Theory, models, and some new findings (pp. 139-159). Boulder, CO: Praeger.

Telzer, E. H. (2011). Expanding the acculturation gap-distress model: An integrative review of research. Human Development, 53, 313-340. https://doi.org/10.1159/000322476

Vu, H. Q., \& Rook, K. S. (2013). Acculturation and intergenerational relationships in Vietnamese American families: The role of gender. Asian American Journal of Psychology, 4, 227-234. https://doi.org/10.1037/a0029750

Weymouth, B. B., Buehler, C., Zhou, N., \& Henson, R. A. (2016). A meta-analysis of parent-adolescent conflict: Disagreement, hostility, and youth maladjustment. Journal of Family Theory \& Review, 8, 95-112. https://doi.org/10.1111/jttr.12126

Wyatt, L. C., Ung, T., Park, R., Kwon, S. C., \& Trinh-Shevrin, C. (2015). Risk factors of suicide and depression among Asian American, Native Hawaiian, and Pacific Islander youth: A systematic literature review. Journal of Health Care for the Poor and Underserved, 26, 191-237. https://doi.org/10.1353/hpu.2015.0059

Yeh, C. J. (2003). Age, acculturation, cultural adjustment, and mental health symptoms of Chinese, Korean, and Japanese immigrant youths. Cultural Diversity and Ethnic Minority Psychology, 9, 34-48. https://doi.org/10.1037/1099-9809.9.1.34

Ying, Y. (2009). Strengthening Intergenerational/Intercultural Ties in Immigrant Families (SITIF): A parenting intervention to bridge the Chinese American intergenerational acculturation gap. In N. Trinh, Y. C. Rho, F. G. Lu, \& K. M. Sanders (Eds.), Handbook of mental health and acculturation in Asian American families (pp. 45-64). New York, NY: Humana Press. https://doi.org/10.1007/978-1-60327-437-1_3

Ying, Y. W., \& Han, M. (2007). The longitudinal effect of intergenerational gap in acculturation on conflict and mental health in Southeast Asian American adolescents. American Journal of Orthopsychiatry, 77, 61-66. https://doi.org/10.1037/0002-9432.77.1.61

\section{Copyrights}

Copyright for this article is retained by the author(s), with first publication rights granted to the journal.

This is an open-access article distributed under the terms and conditions of the Creative Commons Attribution license which permits unrestricted use, distribution, and reproduction in any medium, provided the original work is properly cited. 